\title{
A Study on EMOSPARK-A Revolution in Human Emotion through Artificial Intelligence
}

\author{
Sanghavi Hiremath
}

\begin{abstract}
For as long as we've been imagining emotionally intelligent machines, we have pictured something at least mildly resembling human form. From George Lucas' $C-3 P O$, to the recently-developed Rob kind Zeno R25, our vision for robotic companionship has typically involved two arms and two legs. Taking a different approach is inventor of the Emo Spark console Patrick Levy Rosenthal, who aims to bring artificial intelligence to consumers in the form of a cube small enough to fit in the palm of your hand. Emo Spark is an Android powered Wi-Fi/Bluetooth cube that allows users to create and interact with an emotionally concise intelligence through conversation, music, and visual media. Emo Spark will take not only gaming, but also your TV, smart phone or computer to an entirely different level from anything ever experienced before media. Over time, the cube creates a customized Emotional Profile Graph (EPG) which collects and measures a unique emotional input from the user. The EPG allows the cube to virtually "feel" senses such as pleasure and pain; and "expresses" those desires according to the user. The future of AI interactions is here. Emo Spark is a brand new innovation from Emo Shape, Ltd. that allows you to transfer your real life emotions and desires directly onto a digital platform using state of the art AI measurements and technology. This is the world's first "Emotional Intelligence" device and its capabilities and benefits are unlimited. The Emo Spark uses emotion text and content analysis to measure the emotional responses of several people all at the same time.

Keywords--- Android powered Wi-Fi/ Blue Tooth Cube, Artificial Intelligence, Emotional Profile Graph, Haywire, Webee Automation.
\end{abstract}

\section{INTRODUCTION}

$\mathrm{T}$ HE Emo Spark console is a 90 x 90 x $90 \mathrm{~mm}(3.5 \times 3.5 \times$ 3.5 in) Wi-Fi or Bluetooth enabled cube that interacts with a user's emotions using a combination of content analysis and face-tracking software. In addition to distinguishing between each member of the household, the device uses custom developed technology that Rosenthal says enables it to differentiate between basic human feelings and create emotion profiles of everyone. The Emo Spark console interacts on a conversational level and demonstrates human emotions while it delivers music, games and videos that are the most pleasant to that particular user.

\footnotetext{
Sanghavi Hiremath, Student, Dept. of Computer Science and Engineering, SKSVMACET-Laxmeshwar, India. E-mail:
} sanghavih02@gmail.com

DOI:10.9756/BIJSESC.8250

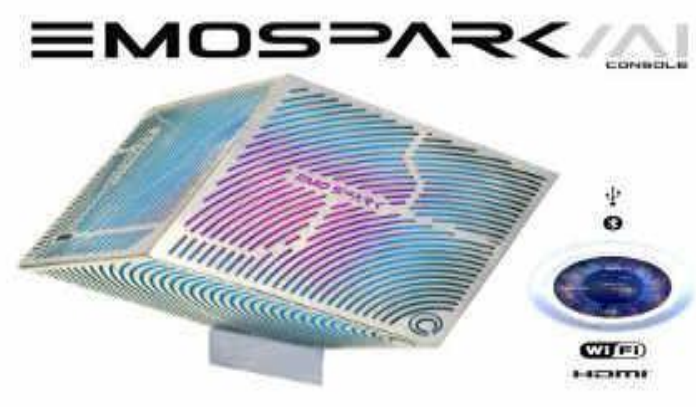

Figure1: MOSPARK

Why emo spark? Aside from direct conversations, the cube can also be spoken to by typing to it via one's (Android) smart phone, tablet, or computer. By providing multiple avenues of communication between user and cube, Emo SPARK can better understand its owners preferences based on eight basic human emotions: joy, sadness, trust, disgust, fear, anger, surprise and anticipation. Artificial Intelligence platforms have come a long way in the past 10 years alone. The "technological singularity" predicted by researchers and commentators is no longer a far-fetched theory, but is rapidly becoming part and parcel of our daily lives. Yet to this day, AI is still viewed as a distant and isolating portent of a technocratic world gone haywire. The time for us to reclaim that singularity is now.

Emo Spark was created with one objective in mind. That is to allow for a true and meaningful understanding between technology and The human emotional spectrum.

\section{HOW DOES IT WORK?}

The human brain processes literally thousands of pieces of information each second - often without consciously realizing it. It registers these physical stimuli as such deceptively simple concepts as sound, motion, and color through a complex model of cognition and reaction; often without our conscious awareness.

Emo Shape, Ltd. designed their Emotion Processing Unit TM (EPU) that enable the user to tap into and register their unique emotional expressions. Since one of the key hallmarks of cognition is based on a consistent emotional response, the EPU literally grows and adapts to the user's responses with each use, enabling a much more broad and realistic emotional reflection. It is designed to become an extension of the user in the digital world. The Emotional Profile Graph can communicate to other AI technologies and allows them to virtually "understand" the user through multimedia, eliciting 
the same emotional response in kind. This response will then accurately convey that expression to other AI technologies.

Each time the user imports or plays media through the Emo Player, they will have the option to rate how it makes them feel and program the cube's EPG to equate that media with an emotional reaction based on the user's EPG. Alternatively, the Emo Player can be used to play back the media and analyze it with direct impact on the EPG of the cube. The cube will also feature a direct interface with Wikipedia, Google Maps and other reference tools for use as a study aid and communication platform.

\section{HARDWARE SPECIFICATION}

As for the hardware and specification of Emo SPARK itself, it is a $90 \mathrm{~mm}$ cube. Inside, aquad-core $1.8 \mathrm{GHz}$ CPU runs Android 4.2.2. Also featured is an "EPU," or Emotional Processing Unit. Android powered, the cube contains $1.8 \mathrm{GHz}$ CPU along with 2 GB of DDR3 memory and Rosenthal's custom-built $20 \mathrm{MHz}$ EPU (Emotion Processing Unit). It has an internal antenna, built-in Wi-Fi $802.11 \mathrm{~b} / \mathrm{g} / \mathrm{n}$ capability and features USB 2.0, Micro USB and HDMI 1.4 ports.

Stretch goals include support for Windows Phone and multi-camera support along with enhanced compatibility for Webee automation. All cubes will have access to a speciallydesigned grid via Emo Shape's servers, where they can meet and interact. Their unique EPG will act like a magnet, attracting other cubes with compatible EPGs. Cubes with similar affinities will connect and share similar media together but the EPG remains secure and private to the cube itself. Only media files can be shared between cubes.

As like our brain Emo Spark can also perceive and process billions of signals and stimuli over time.

1) The Cube connected to Google, Wikipedia and other free resources and is able to answer the project answers in over 39 million topics.

2) You can interact Emo spark by using your Smart phone, Tablet, T.v or PC.

3) Emo SPARK can feel an infinite variety in the emotional spectrum based on 8 primary human emotions, Joy, Sadness, Trust, Disgust, Fear, Anger, Surprise and Anticipation.

4) Emo SPARK learns everything from you, the way you interact with it, comments and responses.

5) This Cube Measure your behavior and emotions, then in turn it will create a emotion profile that make you happy and feel comfortable.

\section{BRIDGING THE GAP}

To communicate with the Android-powered Emo spark, users can simply talk to it through speaking or typing into their tablet, mobile phone (which means it can gauge your emotions on the move), computer or TV. It combines this with facetracking technology to gauge the user's likes and dislikes by categorizing their emotional responses to music, videos and other content (using an emotional spectrum based on seven emotions: joy, sadness, trust, disgust, fear, anger, surprise and
As a concept it's revolutionary and as the complete product where technology and human emotion meet, it is the first of its kind. Nevertheless, one of the key questions being asked, since it is has peaked such interest on the fund raising platform is "just exactly how does it do these things?" Simply put, the device monitors facial expressions and emotions. It can understand conversations. And it knows how to react by offering solutions, and even chats.

\section{WhY MUSIC?}

Emo Spark initially uses music and sound to inform a cube's EPG because music is one of the most direct and immediate stimuli of emotional response. Studies have proven that unborn children can literally "hear" music in utero and react to it.

The Emo Spark Cube uses the same basic principle to experience and register the user's customized data and literally "grow" and adapt to customized audio cues.

At first, sound will be the primary method through which the cube will learn and grow from. The Emo Player will then create a customized EPG for the user that will in turn directly impact the EPG of the cube. Step by step, the cube will use this preliminary sound programming to develop and experience a virtual "life" of its own .

\section{VISUAL INTERACTION}

The Emo Spark can also view a gamer face to face directly in real time on a web cam, observing and responding to various cues. Dedicated plug-ins will recognize those same consistent visual expressions and after receiving a verifiable response, the cube will begin to vicariously experience life with the user. The cube will see when the user has had a difficult day, and express itself sympathetically. The Emo Spark's EPG is color-coded, so the user will be able to recognize the cube's emotional status from its LED lighting. For instance, the user can watch white sparks fly inside the cube's visualization app when it's "in pleasure", and black sparks when it's not.

Emo Spark's app lets the user use a smart device to witness the intensity and nuances of its emotional status in real time at a distance. Emo Spark will then share its reactions with the user via their TV, smart phone or tablet apps. These visualization apps allow the user to see inside the "consciousness" of the cube and monitor what it's "feeling" through its "emotional cloud" and what it's "thinking" through a virtual wall of images and sounds.

\section{COMMUNICATION}

anticipation). Users can also connect with Face book and YouTube to help the cube build up a history of interests. Emospark initially tries to recommend particular pieces of content -- be it a song or a YouTube video -- that might help to improve the user's mood. So, for example, the cube might tell you that your friend Michael has posted a new video onto Face book and it has 12 likes, would you like to watch it. If 
you say yes, the cube will play it on the TV or other device. If you start to laugh, it will show you similar content.

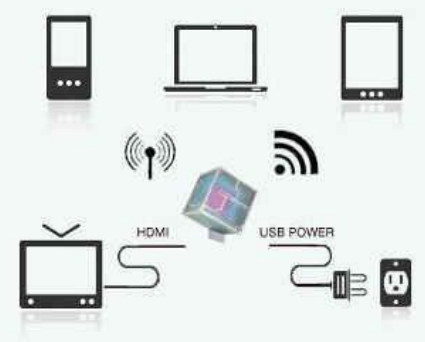

Fguire 2: Interaction of Emo Spark

\section{EMO SPARK APP}

The Emo Spark cube can be accessed remotely through video conferencing facility. The user can interact and engage in conversation with the cube, just like a regular call, through Android's text to speech functionality. He can enter into the space of the cube's "consciousness", exploring the virtual walls of images and information that the cube is linked to -all in real time. Emo Spark cubes connect with one another through social media platforms. Once a reliable EPG is established, an Emo Spark cube can crawl through the web searching for similar or new expressions, and interact with other cubes on a network grid developed by Emo Shape. Over time and experience, the Emo Spark cube can develop a distinctive "personality" of its own, seeking to experience joy and satisfaction -just like humans. This technology will allow the users to literally craft a "life" on to AI technology, becoming greater than the sum of its parts.

\section{APPLICATIONS}

1. The Emo Spark cube also doubles as an e-learning tool.

2. It comes connected to a collection of online

3. knowledge owned by Google, it answer questions on over 39 million topics.

4. It can also be used to control robotic devices, bringing emotional feedback capabilities to a NAO robot or turning a Sphere ball into a virtual pet with its own emotions.

Technically, Emo Spark accesses NASA's MODIS satellite, the Freebase and Wiki databases and results in a platform so innovative it will spin the entertainment world on its side.

\section{CONCLUSION}

Technology that improves our lives is always a priority. Technology that can improve moods and overall happiness is beyond our expectations, yet we are always ready for a break from today's chaotic world.

\section{REFERENCES}

[1] http://photos.prnewswire.com/prnh/20140 106/PH40168-b

[2] www.emospark.com

[3] www.emoshape.com.

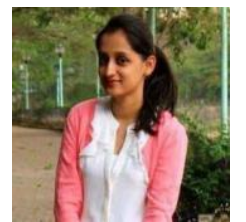

sanghavi Hiremath, $(F, 22)$ Student, Dept. of Computer Science and Engineering, SKSVMACET Laxmeshwar, India. (E-mail:sanghavih02@gmail.com) 